\title{
Recursive Construction of Neural Networks with Long Periodic Behavior ${ }^{1}$ Martín Matamala
}

Laboratoire de l'Informatique du Parallélisme, Ecole Normale Supérieure de Lyon 46, Allée d'Italie, 69364 Lyon Cedex 07, France.

From December 93: Departamento de Ingeniería Matemática, U. de Chile

Casilla 170-correo 3 Santiago, Chile

\section{Extended Abstract}

A neural network of size $n$, is a discrete dynamical system acting on $\Omega_{n}=\{-1,1\}^{n}$, whose transition function, $F_{A}$, is given in term of an $n \times n$ real matrix $A=\left(a_{i j}\right)$ as follows:

$$
\begin{gathered}
F_{A}(x)=\overline{\operatorname{sgn}}(A x) ; \quad(A x)_{i}=\sum_{j=1}^{n} a_{i j} x_{j} \quad i=1, \ldots, n \\
\overline{\operatorname{sgn}}: \mathbb{R}^{n} \rightarrow\{-1,1\}^{n}, \overline{\operatorname{sgn}}(y)_{i}=\operatorname{sgn}\left(y_{i}\right) i=1, \ldots, n \quad \operatorname{sgn}(u)= \begin{cases}1 & u \geq 0 \\
-1 & u<0\end{cases}
\end{gathered}
$$

We are interested in the reverberation neural networks, i.e. neural networks where each state of the system, after a finite number of steps become to himself ( hypercube permutations). We study the question: how many reverberation neural netwoks have really different dynamical behavior?. A related question is asked in [2] where it is defined an equivalence relation whose equivalence class are characterized and the number of elements in any class is proved to be $2^{n} n$ ! where $n$ is the size of the neural network, but nothing is done concerning the number of different class.

Our approach consists in to look the neural networks, in particular reverberation neural networks, as dynamical systems. In order to give a partial answer to our question, we define an equivalence relation which is as the equivalence relation of dynamical systems and we proved, by building recursively $2^{n}$ non equivalent neural networks, that there exists at least $2^{n}$ different class when one considers this relation in the set of the reverberation neural networks of size $n$.

We give a way to build from a threshold function $f: \Omega_{n} \rightarrow \Omega_{1}$ another threshold function $g: \Omega_{n+1} \rightarrow \Omega_{1}$ such that $g$ acts over a vector $(x, u) \in \Omega_{n+1}$, excepte for four vector $y_{1}, y_{2}, y_{3}, y_{4}$, as if only the $n$ first coordinates, $x$, were considered, and then giving the value $f(x)$, moreover, the values $g\left(y_{i}\right), i=1,2,3,4$ are fixed by the construction. So, one can describe the dynamical evolution of $g$ easily in term of those of $f$. Moreover, we show how to obtain threshold functions having an $a$ priori desired behavior.

From these two constructions we give a recursive way for the construction of matrices, i.e., given a matrix $A$ of size $n$ satisfying some hyphotesis we build two matrices $B$ and $C$ of size $n+1$, satisfying also those hyphotesis .

Later, we define an equivalence relation on $P_{n}$, the set of biyective function on $\Omega_{n}$ and we build a function $\eta$ associating to each element in $P_{n}$ a vector of size $2^{n}$. We prove that two function $F$ and $G$ are equivalent iff $\eta(F)=\eta(G)$. Hence, $B$ and $C$ given by the construction define non equivalent neural networks because $\eta\left(F_{B}\right)$ and $\eta\left(F_{C}\right)$ are differents. Later, we prove that given two non equivalent neural networks $A$ and $A^{\prime}$ their extentions are also non equivalent. This fact says that growing the size of the neural networks by one unit, one can double the non equivalent neural netwok number. That explains because we find $2^{n}$ non equivalent neural networks.

Like a corollary, we build a neural network $A$ of size $n$ which has only a cycle of period $2^{n}$.

[1] M.Matamala, (1992)

\section{Reference}

"Recursive Contructions of Periodic Steady State Sequences for Neural Networks". Research Report, Dep. Ingeniería Matemática, Facultad Ciencias Físicas y Matemáticas, Univ. de Chile.

[2] M.Sato. C. Tanaka, (1982)

"Characterization and constructions of Reverberating Networks". Mathematical Biosciences 62:201-217.

\footnotetext{
${ }^{1}$ Partially supported by FONDECYT 0047/90
} 